\title{
Changing Profile of Prostatic Abscess
}

\author{
Suresh K. Bhagat, Nitin S. Kekre, Ganesh Gopalakrishnan, V. Balaji, Mary S. Mathews
}

Department of Urology (SKB, NSK, GG) and Department Clinical Microbiology (VB, MSM), Christian Medical College, Vellore, India

\begin{abstract}
Purpose: To compare the clinical presentation of prostatic abscess and treatment outcome in two different time frames with regards to etiologies, co-morbid factors and the impact of multidrug resistant organism.

Materials and Methods: We retrospectively assessed the charts of 48 patients with the diagnosis of prostatic abscess from 1991 to 2005. The period was divided arbitrarily into two different time frames; phase I (1991-1997) and phase II (1998-2005). Factors analyzed included presenting features, predisposing factors, imaging, bacteriological and antibiotic susceptibility profile, treatment and its outcome.

Results: The mean patient age in phase I $(n=18)$ and phase II $(n=30)$ were $59.22 \pm 11.02$ yrs and $49.14 \pm 15.67$ respectively $(\mathrm{p}=0.013)$. Diabetes mellitus was most common predisposing factor in both phases. Eleven patients in phase II had no co-morbid factor, of which nine were in the younger age group (22 - 44 years). Of these eleven patients, five presented with pyrexia of unknown origin and had no lower urinary tract symptoms LUTS Two patients with HIV had tuberculous prostatic abscess along with cryptococcal abscess in one in phase II. Two patients had melioidotic prostatic abscess in phase II. The organisms cultured were predominantly susceptible to first line antibiotics in phase I whereas second or third line in phase II.

Conclusion: The incidence of prostatic abscess is increasing in younger patients without co-morbid factors. The bacteriological profile remained generally unchanged, but recently multi drug resistant organisms have emerged. A worrying trend of HIV infection with tuberculous prostatic abscess and other rare organism is also emerging.
\end{abstract}

Key words: prostate; infection; abscess; antibiotics; predisposing factors

Int Braz J Urol. 2008; 34: 164-70

\section{INTRODUCTION}

The incidence of prostatic abscess (PA) has declined markedly with the widespread use of antibiotics and the decreasing incidence of urethral gonococcal infections. Predisposing factors for PA include indwelling catheter, instrumentation of lower urinary tract, bladder outlet obstruction, acute and chronic bacterial prostatitis, chronic renal failure, hemodialysis, diabetes mellitus, cirrhosis and more recently, the acquired immunodeficiency syndrome $(1,2)$. The clinical diagnosis of PA has historically been regarded as difficult because of the lack of pathognomonic symptoms or specific clinical signs. With the advent of transrectal ultrasound (TRUS) (3) and computed tomography (CT), the diagnosis of prostatic abscess has been greatly facilitated $(4,5)$.

The pathologic spectrum of PA ranges from microabscesses that resolve with antimicrobial treat- 
ment alone to large multilocular abscesses requiring drainage. Although rare prostatic abscess can result in severe complications, including rupture into the periprostatic space, urethra, rectum (rectourethral fistula), perivesical space, perineum, as well as into the peritoneum and bladder due to either delayed diagnosis or inadequate drainage (6-8).

The spectrum of organisms responsible for the causation of prostatic abscess has changed. In the past, Neisseria gonorrhoeae and Staphylococcus aureus were common (6), nowadays the most common organisms responsible for PA have been gram-negative bacteria, especially Escherichia coli $(1,8,9)$. Recently, we encountered several cases of PA caused by Klebsiella pneumoniae, Entererococci spp, Mycobacteria spp and Burkholderia pseudomallei suggesting the possibility of a shift in the pattern of causation of the disease that prompted us to review the clinical and laboratory data therapeutic details on prostatic abscess over a fourteen-year period.

\section{MATERIALS AND METHODS}

A retrospective study was carried-out on 48 patients with prostatic abscess diagnosed between June 1991 and June 2005. In order to determine changes in disease pattern over time, the 14-year study period was arbitrarily divided into two 7-year periods, phase I (1991 - 1997) and phase II (1998 - 2005). Institutional review board approval is not required for a retrospective study in our country. The factors analyzed were age, presenting features, digital rectal examinations, diagnostic imaging, associated co-morbidity, bacteriological profile, antibiotic susceptibility pattern, treatment modalities and its outcome during each phase. Urine samples were collected as clean catch midstream voided sample and catheter specimen by sterile technique. Pus from prostatic abscess was collected in a sterile culture bottle during transurethral resection of the prostate or ultrasound guided aspiration by aseptic technique. Identification of causative organisms was performed by standard microbiologic methods (10). Antimicrobial susceptibility testing was carried out using disk diffusion method (11). The interpretation was based on the recommendations of
Clinical Laboratory Standards Institute (CLSI) (12). E. coli American Type Culture Collection (ATCC) 25922, P. aeruginosa ATCC 25922 and S. aureus ATCC 27853 were used as quality controls.

Statistical analysis was performed using SPSS (Version 11.0) software. Age was compared using Mann-Whitney U Test between the two phases. Other variables like lower urinary tract symptoms (LUTS), acute urinary retention, pain localization, fever, chills, sepsis, diabetes and digital rectal examination (DRE) findings, were compared between the two phases using Chi-Square Test. All p values less than 0.05 were considered significant. The data are expressed as mean and \pm SD or median and range.

\section{RESULTS}

The baseline data and clinical presentations in both the phases are shown in Table-1. There was a recent statistically significant shift to younger age at presentation $(\mathrm{p}=0.013)$. The clinical presentations in both phases were similar except LUTS and chills (Table-1). DRE measured size, tenderness and induration and revealed similar findings in both phases.

Although the diabetes mellitus was the most common factor in both phases, it was seen less frequently in phase II (53.33\%) than in phase I $(77.77 \%)$ (Table-2). There were four patients with no co-morbidity in phase I. There were 11 patients in phase II with no co-morbid factor, of which nine were in the younger age group (22 - 44 years). Of these 11 patients five presented with pyrexia of unknown origin (PUO) and the cause was prostatic abscess with no LUTS. There were two patients with HIV infection, two with perinephric abscess and one each with chronic liver disease and end stage renal disease in Phase II.

Urine culture was available in 13 of 18 patients in phase I and 28 of 30 patients in phase II (Table-3), it was positive in 9 and 23 respectively. The pus culture was performed in eight patients in phase I and 16 patients in phase II that was positive in two and 14, respectively (Table-3). The urine culture and pus culture were similar in only six cases. The organisms cultured were predominantly susceptible to first line antibiotics (ampicillin, gentamicin, cotrimoxazole 
Table 1 - Clinical presentation.

\begin{tabular}{lccc}
\hline Variables & $\begin{array}{c}\text { Phase I (1991 - 1997) } \\
(\mathrm{N}=18)\end{array}$ & $\begin{array}{c}\text { Phase II (1998- 2005) } \\
(\mathrm{N}=30)\end{array}$ & p Value \\
\hline Mean age in yrs. + SD & $59.22+11.0$ & $49.14+15.7$ & 0.013 \\
N of Patients with & & & \\
$\quad$ LUTS (\%) & $17(94.44)$ & $22(73.33)$ & 0.021 \\
AUR (\%) & $13(72.22)$ & $20(66.67)$ & 0.375 \\
Fever (\%) & $10(55.55)$ & $23(76.66)$ & 0.175 \\
Chills (\%) & $4(22.22)$ & $19(63.33)$ & 0.016 \\
Sepsis (\%) & $4(36.67)$ & $11(36.67)$ & 0.332 \\
\hline
\end{tabular}

$A U R=$ acute urinary tension $;$ LUTS $=$ lower urinary tract symptoms.

Table 2 - Predisposing factors.

\begin{tabular}{lcc}
\hline Variables & $\begin{array}{c}\text { Phase I (1991-1997) } \\
(\mathrm{N}=18)\end{array}$ & $\begin{array}{c}\text { Phase II (1998-2005) } \\
(\mathrm{N}=30)\end{array}$ \\
\hline Diabetes mellitus & 14 & 16 \\
HIV & 0 & 2 \\
Chronic liver disease & 0 & 1 \\
End-stage renal disease & 0 & 2 \\
Perinephric abscess & 0 & 11 \\
Absent co-morbidity & 4 & 2 \\
\hline
\end{tabular}

and quinolones) in phase I whereas organisms were predominantly susceptible to second line (amikacin, ceftazidime) or third line antibiotics (imipenem or meropenem), in phase II. In phase I, of nine isolates four were E. coli and three of them were sensitive to commonly used first line drugs (ampicillin, ciprofloxacin, co-trimoxazole and gentamicin) and one was resistant. In phase II, of nine E. coli isolates only two were sensitive to first line antibiotics (ampicillin, cefuroxime, co-trimoxazole, gentamicin) and rest 7 were resistant and were susceptible to second line (amikacin, ceftazidime) or third line antibiotics (imipenem and meropenem).
Of four Pseudomonas spp two were susceptible to gentamicin and amikacin, and rests were susceptible only to ceftazidime, imipenem and meropenem. Klebsiella spp was susceptible only to ceftazidime, imipenem and meropenem. Burkholderia pseudomallei was susceptible only to ceftazidime, imipenem and meropenem However, the susceptibility of the gram positive organisms remained the same in both the phases.

In phase I diagnosis was confirmed by abdominal ultrasound in nine patients and transrectal ultrasound (TRUS) in three whereas in phase II the common mode of diagnosis was TRUS in 17, trans- 
Table 3 - Bacteriological profile of urine and pus cultures.

\begin{tabular}{lcc}
\hline Culture & $\begin{array}{c}\text { Phase I (1991 - 1997) } \\
(\mathrm{N}=18)\end{array}$ & $\begin{array}{c}\text { Phase II (1998 - 2005) } \\
(\mathrm{N}=30)\end{array}$ \\
\hline Urine & 13 & 28 \\
Escherichia coli & 4 & 9 \\
Staphylococcus aureus & 3 & 2 \\
Pseudomonas aeruginosa & 0 & 4 \\
Klebsiella spp & 0 & 3 \\
Serratia spp & 1 & 0 \\
Citrobacter spp & 0 & 1 \\
Burkholderia pseudomallei & 0 & 2 \\
Fungus & 1 & 2 \\
No Growth & 4 & 5 \\
Culture not available & 5 & 2 \\
Pus & 8 & 16 \\
Escherichia coli & 1 & 6 \\
Staphylococcus aureus & 1 & 3 \\
Pseudomonas aeruginosa & 0 & 12 \\
Ps. aeruginosa + Enterococci & 0 & 1 \\
Klebsiella spp & 0 & 2 \\
Cryptococcus spp & 0 & 1 \\
No growth & 0 & 2 \\
Culture not done & 0 & 2 \\
\hline & & 2 \\
\hline
\end{tabular}

abdominal ultrasound in six (Figure-1) and CT scan in one patient with perinephric abscess and other two with ruptured prostatic abscess making it possible to exactly define the extra-prostatic extent of pus in the ischiorectal fossa and perirectal tissue (Figure-2).

The value of DRE in diagnosing prostatic abscess remained the same in both phases demonstrated by the fact that six patients in phase I and four in phase II were diagnosed based solely on DRE.
As treatment, in addition to appropriate antibiotics, in phase I, 10 patients underwent transurethral resection of the prostate (TURP) along with transurethral drainage of pus, as they were older and with symptoms of prostatic enlargement. Three patients had TUR drainage, four had spontaneous rupture and one patient underwent transperineal aspiration. In phase II, TUR drainage was the most common mode of treatment, which was performed in 14 patients as 


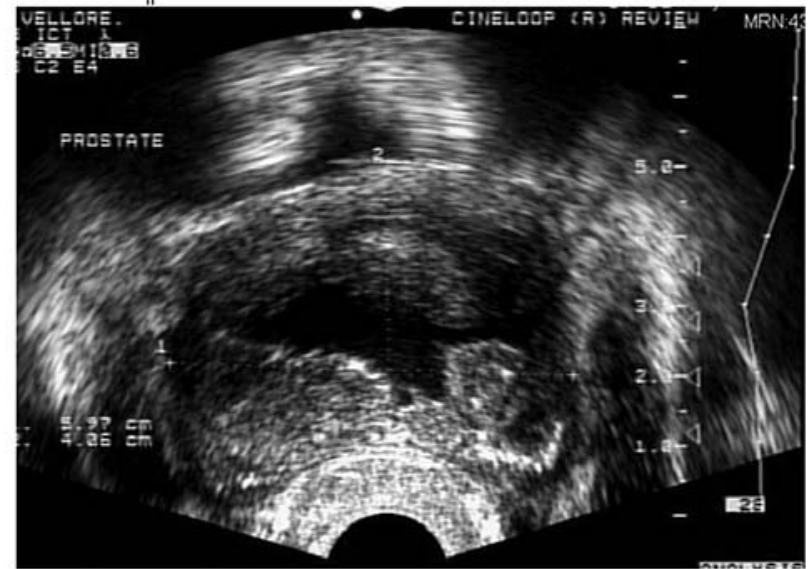

Figure 1 - Transrectal ultrasound showing prostatic abscess.

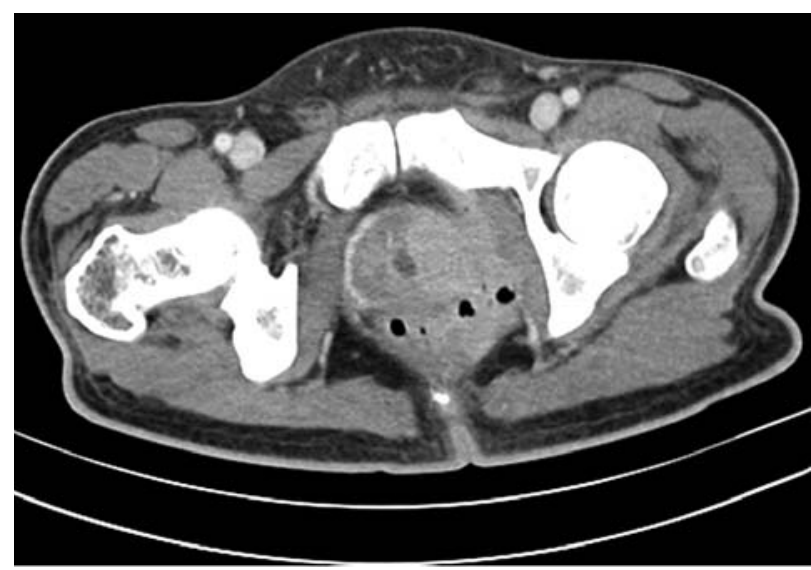

Figure 2-CT scan showing prostatic abscess ruptured into left ischiorectal fossa.

patients were of a younger age group, only four elderly patients with concomitant prostatic enlargement had TURP. Three had TRUS guided aspiration, one with distal penile urethral stricture had transperineal aspiration with statistical process control and four had spontaneous rupture. Two patients with microabscesses and one with melioidosis were treated exclusively with antibiotics. One patient who underwent transperineal aspiration in phase II developed septic shock requiring ventilatory and vasopressure support in intensive care unit. None of the patients in phase I or phase II had septicemia due to formal TURP and TUR drainage.

In phase I, four patients had spontaneous rupture due to delayed diagnosis. One developed perineal abscess and one pararectal abscess requiring open drainage, and in two patients abscesses had ruptured into the prostatic urethra. In phase II, there were four patients with spontaneous rupture due to delayed diagnosis. One developed horse shoe perineal abscess that required open drainage and temporary sigmoid colostomy. One had pararectal abscess that was managed by incision and drainage. One had rectourethral fistula that was treated with antibiotic and suprapubic drainage for three months. In one abscess ruptured into the prostatic urethra. In phase II, there were two patients of HIV infection with tuberculous prostatic abscess, along with tuberculous pyocele and Cryptococcus neoformans isolated on pus culture in one.

All patients recovered well in both the phases except one death in phase II who had melioidosis. Three young patients in phase II following TUR drainage of prostatic abscess developed retrograde ejaculation. Mean duration of hospital stay were similar in both the phases, 11.37 days (range 6 - 23 days) and 9.33 days (range 2 - 28 days ) as was the duration of antibiotic therapy 28 days ( $14-42$ days) and 30 days (9 - 90 days) in phase I and phase II respectively.

\section{COMMENTS}

Prostatic abscess is an infrequent condition in the modern antibiotic era with an incidence of $0.5 \%$ to $2.5 \%$ of all prostatic disease (8). Prostatic abscess can occur in patients of any age but is mainly found in men in their $5^{\text {th }}$ and $6^{\text {th }}$ decade of life (13). As seen in our series, prostatic abscess is occurring in a younger age group.

Predisposing factors for development of prostatic abscess are diabetes mellitus, bladder outlet obstruction, indwelling catheter, chronic renal failure, patients on hemodialysis, chronic liver disease and more recently HIV infection (14). In our series, diabetes was the most common predisposing factor, with HIV causing tuberculous abscesses, in two patients. In phase II $53 \%$ of patients were diabetic. They were younger and keeping with the WHO report (15) of diabetes occurring in younger individuals in the Indian subcontinent. Three patients $(21.42 \%)$ in phase I and 7 patients $(43.75 \%)$ in phase II were diagnosed to be diabetic for the first time when they presented with 
prostatic abscess. This could be a major new form of presentation in keeping with the increased incidence of diabetes.

Prostatic abscess should be considered as a possible etiology when evaluating for PUO in younger men as five of 11 patients without predisposing factor presented with PUO.

The clinical diagnosis of prostatic abscess is sometimes difficult because of nonspecific symptoms (8). This condition usually presents as an irritative voiding symptoms, perineal pain, and fever and occasionally as acute urinary retention (1). In our series, 17 patients $(94.44 \%)$ in phase I and 22 patients $(73.33 \%)$ in phase II presented with irritative LUTS. This may be due to the fact that patients were of the older age group in phase I than in phase II. The patients with prostatic abscess presented more commonly with fever and chills in phase II than in phase I. The number of patients with sepsis was higher in phase II $(36.67 \%)$ than in phase I $(22.2 \%)$. This is most probably due to infection caused by multi drug resistant bacteria related to the misuse of antibiotics in the community. In our series in phase I, $75 \%$ of E. coli were sensitive to commonly used first line drugs (ampicillin, ciprofloxacin, co-trimoxazole and gentamicin) and in phase II, more than $75 \%$ of E. coli were resistant to first line antibiotics and were susceptible to second line (amikacin, ceftazidime) or third line antibiotics (imipenem and meropenem).

The microbiology of prostatic abscess has undergone a complete metamorphosis in the antibiotic era. More recently, various reports have shown that the common organisms causing prostatic abscess are E. coli and other enteric gram negative bacilli $(1,8,9)$. More recently we have reported two cases of prostatic abscess due to Burkholderia pseudomallei (16).

However, the prevalence of immunocompromised individuals has increased in the modern era (phase II), and the potential for uncommon fastidious pathogens, particularly mycobacterial, fungal and anaerobic pathogens, melioidosis, in addition to typical gram-negative bacilli, will make the diagnosis of prostatic abscess more complicated $(14,16,17)$.

Surprisingly urine culture and pus culture isolates were similar in only six cases (all in phase II). Of these, 4 were gram negative bacilli, this includes $\mathrm{E}$. coli $(n=2)$, Klebsiella spp $(n=1)$ and Pseudomonas $\operatorname{spp}(\mathrm{n}=1)$ and 2 were gram positive cocci (S. aureus). Of six patients, five were diabetic and four had sepsis at presentation. It is important to send material for culture (pus, urine, and/or prostatic chips) in order to identify the etiologic agent, especially in immunocompromised patients because they usually present with uncommon microorganisms (18). Urine culture may be negative unless the abscess ruptures into urethra or bladder. Thus it is important to emphasize that pus culture and sensitivity should be performed routinely for management of prostatic abscess.

Although the bacteriological profile was similar in both phases, it is important to note that the antibiotic susceptibility profile had changed, with organisms resistant to first line drugs and sensitive only to higher antibiotics.

In our series, trans-abdominal USG was the most common modality of diagnosis in phase I, but in phase II, TRUS became the major diagnostic tool and was performed in $56.67 \%$ of patients and has now become a standard protocol as the transrectal probe was acquired later part of $1^{\text {st }}$ phase.

Prostatic abscess currently occurring in a relatively younger population has treatment implications. Transurethral drainage could result in retrograde ejaculation as seen in three patients in this series and hence one would like to resort to transperineal / transrectal aspiration. TURP is indicated in elderly patients with associated bladder outlet obstruction due to prostatic enlargement. In our series, in phase I most of the patients being older with associated obstructive LUTS had a formal TURP, in addition to drainage and the abscess. In phase II, $50 \%$ of patients were treated by transurethral drainage of abscess, 3 patients had TRUS guided aspiration where one required TUR drainage due to recurrent prostatic abscess. In very few cases, open surgical drainage may be indicated mainly in those patients with extraprostatic involvement (17). In this series two patients with spontaneous rupture in each phase required open surgical drainage.

Potential complications due to a late diagnosis include spontaneous rupture into the urethra, perineum, bladder or rectum and the development of septic shock with a mortality rate of $1 \%$ to $16 \%(8)$. There was one mortality due to infection with melioidosis in this series. 


\section{CONCLUSIONS}

Prostatic abscess should be considered in the differential diagnosis of young men who present with pyrexia of unknown origin. It could be the primary presentation in a recently diagnosed diabetic. The incidence of prostatic abscess is increasing in younger males. This is probably related to the higher incidence of diabetes in younger males in this region. Clinical findings could be subtle especially in younger men who may not present with LUTS. While the bacteriology remains largely unchanged, the emergence of multi drug resistant organisms points to the rampant misuse of antibiotics. The emergence of HIV brings the added concern that some of the abscesses could be the result of tuberculous infection.

\section{CONFLICT OF INTEREST}

None declared.

\section{REFERENCES}

1. Angwafo FF 3rd, Sosso AM, Muna WF, Edzoa T, Juimo AG: Prostatic abscesses in sub-Saharan Africa: a hospital-based experience from Cameroon. Eur Urol. 1996; 30: 28-33.

2. Leport C, Rousseau F, Perronne C, Salmon D, Joerg A, Vilde JL: Bacterial prostatitis in patients infected with the human immunodeficiency virus. J Urol. 1989; 141: 334-6.

3. Cytron S, Weinberger M, Pitlik SD, Servadio C: Value of transrectal ultrasonography for diagnosis and treatment of prostatic abscess. Urology. 1988; 32: 454-8.

4. Davidson KC, Garlow WB, Brewer J: Computerized tomography of prostatic and periurethral abscesses: 2 case reports. J Urol. 1986; 135: 1257-8.

5. Vaccaro JA, Belville WD, Kiesling VJ Jr, Davis R: Prostatic abscess: computerized tomography scanning as an aid to diagnosis and treatment. J Urol. 1986; 136: 1318-9.

6. Sargent JC, Irwin R: Prostatic abscess: a clinical study of 42 cases. Am J Surg. 1931; 11: 334-7.

7. Mitchell RJ, Blake JR: Spontaneous perforation of prostatic abscess with peritonitis. J Urol. 1972; 107: 622-3.
8. Granados EA, Riley G, Salvador J, Vincente J: Prostatic abscess: diagnosis and treatment. J Urol. 1992; 148: 80-2.

9. Jacobsen JD, Kvist E: Prostatic abscess. A review of literature and a presentation of 5 cases. Scand J Urol Nephrol. 1993; 27: 281-4.

10. Myer's and Koshi's Manual of Diagnostic Procedure in Medical Microbiology and Immunology/Serology. Faculty, Department of Clinical Microbiology, Christian Medical College, Vellore, India. Pondicherry, All India Press, 2001.

11. Bauer AW, Kirby WM, Sherris JC, Turck M: Antibiotic susceptibility testing by a standardized single disk method. Am J Clin Pathol. 1966; 45: 493-6.

12. Clinical Laboratory Standards Institute (formerly, National committee for Clinical Laboratory Standards. Performance Standards for Antimicrobials Disc Susceptibility Tests. 8th ed. Approved Standards NCCLS Document M2-A7, Wayne, PA: National Committee for Clinical Laboratory Standards, 2000.

13. Pai MG, Bhat HS: Prostatic abscess. J Urol. 1972; 108: 599-600.

14. Trauzzi SJ, Kay CJ, Kaufman DG, Lowe FC: Management of prostatic abscess in patients with human immunodeficiency syndrome. Urology. 1994; 43: 629-33.

15. Mohan V, Deepa M, Deepa R, Shanthirani CS, Farooq $\mathrm{S}$, Ganesan A, et al.: Secular trends in the prevalence of diabetes and impaired glucose tolerance in urban South India--the Chennai Urban Rural Epidemiology Study (CURES-17). Diabetologia. 2006; 49: 1175-8.

16. Viswaroop BS, Balaji V, Mathai E, Kekre NS: Melioidosis presenting as genitourinary infection in two men with diabetes. J Postgrad Med. 2007; 53: 108-10.

17. Ludwig M, Schroeder-Printzen I, Schiefer HG, Weidner W: Diagnosis and therapeutic management of 18 patients with prostatic abscess. Urology. 1999; 53: 340-5.

18. Oliveira P, Andrade JA, Porto HC, Pereira Filho JE, Vinhaes AF: Diagnosis and treatment of prostatic abscess. Int Braz J Urol. 2003; 29: 30-4.

\section{Accepted after revision:}

March 6, 2008 